\title{
DEUTSCHE UND POLNISCHE SPRICHWÖRTER IN ARTIKELÜBERSCHRIFTEN VOR DEM HINTERGRUND IHRES BEKANNTHEITSGRADES
}

0. „Erst in der Verwendung lebt das Sprichwort: in der Sammlung ist es tot" diese Worte Samuel Singers (Singer 1944-1947: 145, Bd. 3; zit. nach Mieder 2004: 417) haben Jahre später eine Reihe von Untersuchungen zu Textfunktionen des Sprichworts ausgelöst, die eigentlich bis heute anhalten, nur die analysierten Textsorten und Untersuchungsaspekte ändern sich. Lange Zeit wurden Sprichwörter in erster Linie vor allem in mündlicher Kommunikation und in der Schrift in literarischen Texten thematisiert. Mit der Zeit verlagerte sich der Schwerpunkt auf Texte und Kommunikation, die die beiden Aspekte der Mündlichkeit und der Schriftlichkeit in gewisser Weise komplementär in sich vereinigen: die Sprache der Massenmedien. Auch die Sprache der Printmedien weist - obwohl schriftlich - bestimmte Merkmale der Mündlichkeit auf, was aus den spezifischen kommunikativen Bedingungen resultiert, u. a. daraus, dass in journalistischen Texten immer häufiger Elemente der Umgangssprache verwendet werden, um so bei einem breiteren Lesepublikum anzukommen.

In den Printmedien scheinen die Sprichwörter eine neue Blütezeit zu erleben. Zum einen betrifft das die Sprache der Presse, zum anderen die der Werbeanzeigen. Sprichwörter sind an verschiedenen Stellen unterschiedlicher Texte anzutreffen, im Folgenden soll ihr Vorkommen in Artikelüberschriften thematisiert werden.

1. Überschriften erfüllen wie alle Titel und auch Schlagzeilen in Werbeanzeigen mehrere Funktionen in Bezug auf den Text, dem sie vorausgehen. Rein technisch gesehen haben sie die Aufgabe, konkrete Texte voneinander zu unterscheiden, weshalb sie immer graphisch durch größeren Fettdruck abgehoben sind, und dienen erstmal „zur Identifizierung des Textes“ (Nord 1993: 27). Diese Funktion teilen sie mit Eigennamen und werden daher von manchen Forschern zu Eigennamen gerech- 
net, wogegen andere (vgl. u.a. Hellwig 1984: 7f.) mehrere Gegenargumente vorbringen. Sie stellen jedenfalls den ersten Kontakt zum Leser her und sollen ihn auf den eigentlichen Text aufmerksam machen, sein Thema und seinen Inhalt so ankündigen, dass beim potenziellen Leser Interesse geweckt und er zum Lesen angeregt wird. Diese Funktion steht der der Werbeschlagzeilen nahe, daher werden Überschriften auch als Schlagzeilen bezeichnet (vgl. u.a. Sandig 1971, 1986). Um diese Funktion erfüllen zu können, soll die Überschrift einerseits durch ihre besondere, attraktive und originale Formulierung auffallen, andererseits kurzgefasste Vorinformationen über den Inhalt des Artikels als „eine schnelle Orientierung“ (Nord 1993: 103) liefern. Diese Vorinformation kann zugleich einen vorausgreifenden Kommentar mitenthalten, der den nachfolgenden Inhalt verstehen bzw. interpretieren hilft. Da die Überschriften möglichst kurzgefasst sein müssen, wird bei längeren und wichtigeren Artikeln von Ober- und Unterzeilen Gebrauch gemacht, so dass die ersten als sog. ,primär anregenden Schlagzeilen“ (Di Meola 1998: 21) die Reizfunktion übernehmen und als „Attraktivmacher“ (Sandig 1986: 228) dienen, während die zweiten als „transparente primäre informative Schlagzeilen“ (Di Meola 1998: 21) sachliche Verweise auf den Inhalt des Ko-Textes enthalten.

Damit Überschriften ihre Funktionen gut erfüllen können, werden sie ähnlich wie Werbeschlagzeilen nicht selten von Fachleuten nach bestimmten Regeln kreiert. Die Aufmerksamkeit in einem Pressetext können Elemente erregen, die einerseits als bekannt, das heißt im Gedächtnis schon verankert, identifiziert werden, andererseits als irgendwie verändert wirken. Als solche kommen in erster Linie alle konventionalisierten Formulierungen, darunter Phraseologismen und Sprichwörter, als „sprachliche Fertigware“ (Mieder 2004: 46) in Frage. Sprichwörter in Überschriften dienen laut Lüger (1995: 36) ,der Lesewerbung, fördern die Anschaulichkeit, und bilden gleichzeitig rezeptionssteuernde Signale“. Umurova (2005: 108) stellt fest, dass aus Sprichwörtern bestehende Titel in ihrem Korpus alle für einen Titel erforderlichen Merkmale aufweisen wie: „Einzigartigkeit, Kürze, Elliptik, Nominativität, Verwendung von Reizwörtern, Neuheit, Tradition, Sicherheit, Verbreitung, Aufforderung zum Handeln, Informativität, Provokation und Irritation". Daraus geht auch hervor, dass die meisten Sprichwörter im Titel in modifizierter Form auftreten.

Modifikationsverfahren, denen die Sprichwörter unterzogen werden, sind für jede Sprache identisch, doch die dabei benutzten ausgewählten Einzelverfahren können anders sein, abhängig von der Textsorte und der kommunikativen Absicht einerseits und von den einzelsprachlichen intralingualen Relationen im gegebenen Text andererseits. Inhaltlich und formal, d.h. bezogen auf die strukturelle Sprichwortspezifik wie Zweigliedrigkeit, Reim, Rhythmus, Alliteration, können jedoch ähnliche Resultate wie u.a. Überraschungseffekte oder Humor erzielt werden. Wegen der einzelsprachspezifischen Grundlagen und Modifikationsbedingungen sind sprachspielerische Abwandlungen und damit auch die sog. Antisprichwörter meist nicht übersetzbar. Eine kontrastive Untersuchung der Modifikationen besteht dem- 
nach vor allem darin, zu zeigen, welchen Modifikationstechniken welche Sprichwörter am häufigsten unterzogen werden.

2. Bei der Wahl der Sprichwörter für Artikelüberschriften sind die allgemeinen Funktionen der Überschriften, die Thematik des Beitrags und der Bezug des gewählten Sprichwortes darauf, sein Bekanntheitsgrad und die formal-inhaltlichen Möglichkeiten seiner Abwandlung zu berücksichtigen. Der Bekanntheitsgrad scheint dabei kein unwichtiger Faktor zu sein, weil nur bekannte Sprichwörter ihr Wiedererkennen in der wie auch immer veränderten Form und die sprachspielerische Rezeption sichern können. In der Konsequenz gibt es unter den Sprichwörtern, wie unter allen Phraseologismen, Sprüchen und Zitaten, solche, die häufiger bzw. seltener als Material für Schlagzeilen in Frage kommen. Dabei scheint die strukturelle Beschaffenheit der Sprichwörter wichtiger zu sein als ihr inhaltlicher Gehalt, der ja durch die vorgenommenen Modifikationen und den Bezug zum nachfolgenden Ko-Text ohnehin meist verändert wird. Unverändert bleibt die in Sprichwörtern enthaltene Verallgemeinerung gewisser kollektiver Erfahrungen und das feste Bildungsmuster - zwei Eigenschaften, die ihre Eignung und Beliebtheit in Überschriften erklären. Sprichwörtliche Bildungsmuster sind nicht stark differenziert, die üblichsten Formen sind: Wie/Wer/Was $x$, so/der/das $y$; $x$ ist/hat $y$; ohne $x$ kein $y$ / $j a k / k t o / c o x$, to/ten $y ; x$ to/ma y (vgl. Beispiele unten).

Wolfgang Mieder hat in zahlreichen Untersuchungen zur textuellen Verwendung der Sprichwörter (vgl. u.a. Mieder 1973, 1992, 1997, 2004) deutsche und englische bzw. amerikanische Sprichwörter züsammengestellt, die am häufigsten in Texten aller Art vorkommen, den meisten Modifikationen unterliegen und Basis für die sog. Antisprichwörter ergeben. Laut der neuesten Untersuchung von Litovkina und Mieder (2006: 12ff.) gehören zu den populärsten medialen Sprichwörtern das deutsche Morgenstunde hat Gold im Munde und das englische Old soldiers never die (they simply fade away). Darüber hinaus haben sie, gestützt auf weitere ähnliche einzelsprachliche Untersuchungen u. a. zum Russischen, Französichen, Ungarischen, festgestellt, welche international verbreiteten Sprichwörter in mehreren Sprachen sich vergleichbarer Beliebtheit als Modifikationsbasis erfreuen. In diesen Untersuchungen ist die polnische Sprache nicht mitberücksichtigt worden. Der Grund dafür scheint u.a. darin zu liegen, dass alle genannten Analysen auf erstellten Sprichwortminima basieren und solche für das Polnische eigentlich noch nicht vorliegen. Für das Deutsche sind bisher mindestens drei nach verschiedenen Kriterien und Methoden erstellten Minima vorgelegt worden, darunter eins mit 15 Sprichwörtern (vgl. Mieder 1992: 16), eins mit 57 (vgl. Baur/Chlosta 1996: 22) und das neueste mit 20 (vgl. Kim 1999, nach Mieder 2004: 44f.).

Das erste polnische von Szpila (vgl. Szpila 2002: 41f.) erstellte Minimum umfasst 72 Sprichwörter, aufgelistet, wie die deutschen, in absteigender Reihenfolge der Bekanntheit. Auch wenn sich gewisse Vorbehalte gegenüber dieser Liste erheben ließen, so ist sie im Moment die einzige verfügbare, die man mit den deutschen 
Minima vergleichen kann und wird daher im Weiteren berücksichtigt. Dem unten angeführten neuesten deutschen Sprichwortminimum von Kim (1999, zit. nach Mieder 2004: 44f.) wurden polnische analoge Sprichwörter aus dem Minimum von Szpila beigefügt, versehen in Klammern mit der Nummer, unter der sie dort verzeichnet sind. Darüber hinaus verweist der Buchstabe nach dem deutschen Sprichwort kurz auf die Herkunft der Sprichwörter: $B=$ Bibel, $L / G=$ Latein/Griechisch. Die Zusammenstellung zeigt große Übereinstimmungen in der Popularität der Sprichwörter beider Sprachen und auch ihre weitgehende gemeinsame Herkunft, deren Quellen vor allem in der Bibel und antiken griechischen und lateinischen Texten liegen. Auf die Differenzen bezüglich der Stelle in den Listen soll wegen der Unterschiede in den Erhebungmethoden nicht näher eingegangen werden. Nur in fünf Fällen gehören die polnischen analogen Sprichwörter nicht zum polnischen Minimum: $6,9,12,15,16$, auch wenn sie, rein subjektiv gesehen, dem polnischen Benutzer nicht unbekannt sind und in allen polnischen wie auch polnisch-deutschen und deutsch-polnischen Sprichwörterlexika verzeichnet sind. Von diesen fünf sich nicht deckenden Sprichwörtern weisen nur drei $(9,12,15)$ keine gemeisame Herkunft auf.

1. Wer andern eine Grube gräbt, fällt selbst hinein. B Kto pod kim dołki kopie, (ten) sam w nie wpada. (3)

2. Morgenstunde hat Gold im Munde. L Kto rano wstaje, temu Pan Bóg daje. (4)

3. Der Apfel fällt nicht weit vom Stamm. G/L Niedaleko pada jabłko od jabłoni. (24)

4. Lügen haben kurze Beine. $\mathbf{L}$ Kłamstwo ma krótkie nogi. (60)

5. Reden ist Silber, Schweigen ist Gold. B Mowa jest srebrem, a milczenie złotem. (45)

6. Was du heute kannst besorgen, (das) verschiebe nicht auf morgen. G/L Co masz zrobić dziś, nie odkładaj do jutra. (-)

7. Wer zuletzt lacht, lacht am besten.

Ten się śmieje najdłużej, kto się śmieje ostatni. (34)

8. Man soll den Tag nicht vor dem Abend loben. L Nie chwal dnia przed zachodem słońca. (49)

9. Ehrlich währt am längsten. Uczciwość najbardziej popłaca. (-)

10. Besser den Spatz in der Hand als die Taube auf dem Dach. $\mathbf{L}$ Lepszy wróbel w garści niż gołąb na dachu. (2)

11. Ohne Fleiß kein Preis. L Bez pracy nie ma kołaczy. (9)

12. Wer im Glashaus sitzt, soll nicht mit Steinen werfen. Kto siedzi w szklanym domu, niech nie rzuca kamienami. (-) 
13. Was Hänschen nicht lernt, lernt Hans nimmermehr.

Czego Jaś się nie nauczy, tego Jan nie będzie umiał. (14)

14. Was du nicht willst, das man dir tu', das füg' auch keinem andern zu. L

Nie czyń drugiemu, co tobie niemiłe. (16)

15. Wer den Pfennig nicht ehrt, ist des Talers nicht wert.

Kto nie szanuje małego, ten niewart dużego. (-)

16. Es ist noch kein Meister vom Himmel gefallen. $\mathbf{L}$

Nikt się mistrzem nie rodzi. (-)

17. Wie man in den Wald schreit, so schallt es heraus.

$\approx$ Jak Kuba Bogu, tak Bóg Kubie.// Jak do lasu wołasz, tak ci się odezwie. (7)

18. Einem geschenkten Gaul schaut man nicht ins Maul. G/L

Darowanemu koniowi nie zagląda się w zęby. (19)

19. Wer zu spät kommt, den bestraft das Leben. L

Kto późno przychodzi, sam sobie szkodzi. (69)

20. Der Krug geht solange zum Brunnen, bis er bricht. $\mathbf{L}$

Dopóty dzban wodę nosi, dopóki się ucho nie urwie. (17)

Um festzustellen, ob in Artikelüberschriften wirklich bekannte Sprichwörter genutzt werden, sind die gefundenen Belege mit den entsprechenden Minima verglichen und in einem weiteren Schritt darauf überprüft worden, ob die nicht modifizierten und die modifizierten Sprichwörter diesbezüglich einen Unterschied aufweisen, wobei die kurze erste Analyse weiterer Verifizierungen bedarf und keinen Anspruch auf verbindliche Aussagen erheben kann.

3. In Bezug auf das exzerpierte deutsche und polnische Material lässt sich zunächst festhalten, dass mehr als die Hälfte aller in den Überschriften benutzten Sprichwörter in den Minima und auch in der o.g. Zusammenstellung vertreten ist, was die aufgestellte These zwar nicht zwingend bestätigt, aber untestützt. Interessant ist, dass die verwendeten Sprichwörter meistens nicht mir einmal, sondern gleich mehrmals die Basis für Überschriften zu Artikeln mit recht unterschiedlicher Thematik bilden. Da auf die Art der vorgenommenen Modifikation nicht eingegangen wird, wird in den Belegen auf die Angabe der Thematik der einzelnen Artikel zum Teil verzichtet.

Im analysierten Material dominiert unter den deutschen Sprichwörtern eindeutig Reden ist Silber, Schweigen ist Gold, und zwar nur in modifizierter Form. In einem Beleg wurde zwar der Wortlaut nicht geändert, dafür aber der Sprechakt, indem die sprichwörtliche Feststellung zu einer Frage wurde, die den nachfolgenden Inhalt anzweifeln oder in Frage stellen kann, hier dagegen signalisiert, dass es für das angesprochene Problem keine allgemeingültige Lösung gibt. Die Sprechaktänderung gehört im Falle der Sprichwörter zu den eher selten genutzten Modifikationen, möglich ist sie dennoch, weil das Sprichwort seine kommunikative Funktion erst im konkreten Kontext erhält (vgl. Mieder 2004: 41). 
- Reden ist Silber, Schweigen ist Gold? (DS 28/08) - [Sollen Ärzte einem todkranken Patienten die Wahrheit sagen oder nicht]

Die weiteren Belege weisen das Sprichwort in substituierter, erweiterter, reduzierter und einer kombinierten, reduzierten und zugleich substituierten Form auf.

Reduziert wird im Allgemeinen seltener der erste, häufiger der zweite Teil, weil dadurch eine Art Spannung ausgelöst wird, die zum Lesen des Artikels anregen soll, um in dem Artikelinhalt die Lösung zu finden. Bei der Substitution wird gern die phonetische und/oder morphologische Ähnlichkeit zwischen dem substituierten und substituierenden Element zum Erzielen sprachspielerischer Effekte genutzt (vgl. Prędota 1996: 30). Darüber hinaus besteht in allen Fällen ein inhaltlicher Bezug zum Artikel, der nicht selten durch eine wiederholte Verwendung des Sprichwortes an verschiedenen Stellen des Ko-Textes, sei es am Anfang oder im weiteren Textverlauf, verdeutlicht wird. Stilistisch kann die durch das halbreduzierte Sprichwort geöffnete Spannungsklammer geschlossen werden, indem auf das im Titel angeführte Sprichwort am Ende der Ausführung Bezug genommen und es in seiner vollen Form verwendet wird (vgl. Belege unten):

- Reden ist Silber, Reklame ist Gold. (S 42/97) [Telefonieren zum Billigtarif mit Werbung dazwischen]

- Schweigen ist Gold (DS 23/02) [Befreite Geiseln sagen nichts zum erlebten Banküberfall]

- Schweigen ist Geld (S 44/00) [Untertitel: Mit unbedachten Äusserungen brachte Europa-Banker Wim Duisenburg den Euro weiter ins Trudeln]

Mehrmals belegt in fast immer modifizierter Gestalt ist auch das an erster Stelle des Minimums von Kim (1999) plazierte Sprichwort Wer andern eine Grube gräbt, fällt selbst hinein, des Weiteren die Positionen 2, 7, 10, 18, 19.

- Wer anderen eine Nase dreht... (DS 47/02) [Die Berliner Aufführung der Oper Die Nase von D. Schostakowitsch]

- Wer zuletzt lacht... (TVT 18/07) [Vorstellung der bekanntesten deutschen Komiker]

- Wer zu spät kommt, den bestraft der Euro (S 18/98) [Lange Vorbereitung deutscher Unternehmen auf die Umstellung auf den Euro]

Im polnischen Material sind, ähnlich wie im Deutschen, seltener in nicht modifizierter, häufiger in oft mehrfach modifizierter Form folgende Sprichwörter aus dem polnischen Minimum von Szpila (2002: 41f.) mehrmals vertreten:

- Milczenie nie zawsze jest ztotem (GW 75/01) [Der Finanzminister schweigt und antwortet nicht auf die Vorwürfe der Presse.]

- Nie taki kruk straszny, jak go myśliwi maluja (GW 186/07) [Wissenschaftler behaupten, dass Raben für das Kleinwild keine Gefahr sind, wie die Jäger es sehen] 
- Nie taki diabel straszny (GW 185/07) [Ergebnisse einer Umfrage nach der Toleranz der Polen gegenüber Fremden] Letzter Satz des Artikels: Diabeł w żadnej części nie jest taki straszny, jak go od wielu pokoleń malowano.

- Nie taki Glódź straszny (GW 88/08) [Diskussion zu der Ernennung des Erzbischofs zum Metropoliten von Gdańsk]

- Nie taki papież straszny (GW 34/08) [Erläuterung der konservativen Ansichten von Papst Benedikt XVI.]

- Nie taki muzutmamin straszny (GW 45/07) [Vorurteile im Einschätzen von Menschen anderen Glaubens]

- Maqdry po szkodzie (WObr. 21/07) [Erst nach spektakulären Unfällen haben die Organisatoren die Sicherheitsbedingungen auf den Rennbahnen verbessert]

- Mądry Czech po szkodzie (W 16/01) [die tschechische Wirtschaft nach der Währungskrise]

- Polak lubi być madry po szkodzie (GW 223/06) [Die Währungskrise in Ungarn wird keine Lehre sein für polnische Finanzinstitutionen, weil die Polen die negativen Folgen falscher Entscheidungen selbst spüren müssen]

- Niedostateczna kapitalizacja, czyli madry fiskus po szkodzie (GW 19/05) [Nach dem EU-Beitritt sind die ausländischen Steuerzahler durch die Vorschriften benachteiligt]

- Jaki pies, taki pan (N 51-52/98) [Untertitel: Co twój pupil mówi o tobie]

- Jaki kraj, taki boom (GW 75/04) [In einem nicht besonders reichen Land kann das Wirtschaftswachtum auch nicht gleich sehr hoch sein]

Nicht gefunden in dem analysierten Material wurden vom deutschen Minimum die Positionen 6, 9, 12, 16, 17, auf der polnischen Seite 6, 8, 9, 12, 15, 16, darunter auch alle Sprichwörter, die im polnischen Minimum nicht auftreten.

4. Da die Sprichwortminima als gezielte Zusammenstellungen eher nicht allgemein bekannt sind, kann das Auftreten der in ihnen verzeichneten Sprichwörter in Artikelüberschriften eine Art Bestätigung für ihre Bekanntheit sein. Andererseits sollte jedoch überprüft werden, ob und welche Sprichwörter in Überschriften genutzt werden, die nicht in die Minima aufgenommen wurden. Dabei sollen, um wie beim Erstellen der Minima eine Zufallswahl auszuschließen, nur mehrmals verwendete Sprichwörter beachtet werden. Bezogen auf das Minimum von Kim können folgende deutsche Sprichwörter genannt werden, die allerdings alle in einem der beiden früheren Minima (vgl. Mieder 1992: 16 und Baur/Chlosta 1996: 22) verzeichnet sind:

- Ende gut, alles gut.

- Ende gut-alles gut? (S 29/02)

- Jacke gut, alles gut (S 41/01)

- Ein Unglück kommt selten allein. 
- Ein Hammer kommt selten allein (S 1/99)

- $\quad$ Eine Affäre kommt selten allein (S 5/00)

- Zeit ist Geld.

- Zeit ist Geld-mehr oder weniger (S 48/02)

- $\quad$ Zeit ist Leben (S 8/04)

Im Polnischen gehören zur Gruppe der vom Minimum nicht erfassten, aber als Überschriften relativ oft verwendeten Sprichwörter u.a. folgende:

- Jak trwoga, to do Boga.

- Jak trwoga, to do Boga (A 17/08)

- Jak trwoga, to do wroga (GW 16/02)

- Jak trwoga, to do Napoleona (P 12/06)

- W zdrowym ciele zdrowy duch.

- $\quad W$ zdrowym ciele zdrowy duch (A 1/08)

- $\quad W$ zdrowym ciele chory duch (W 20/01)

- $\quad W$ zdrowym ciele zdrowy polityk (W 50/04)

- Diabeł tkwi w szczegółach.

- Diablo tkwi w szczególach (W 28/02)

- Diably tkwia w szczególach (A 10/07)

- $\quad$ Diabel w szczególach (P 20/07)

- Co za dużo, to niezdrowo.

- Co za czysto, to niezdrowo (W 30/04)

- Co za dużo... (W 46/01)

- Kto daje i odbiera, ten się po piekle poniewiera.

- Kto daje i odbiera... (W 30/07)

- Kto daje, kto zabiera... (P 39/06)

- Kto daje i odbiera... (GW 54/08

- $\quad$ Nie od razu Kraków zbudowano.

- $\quad$ Nie od razu Kraków wysadzano (W 38/99)

- $\quad$ Nie od razu Kraków (GW 196/01)

- Jak nie kijem, to pałą.

- Jaknie VAT-em je, to... VAT-em (GW 9/01)

- $\quad$ Nie kijem, to z procy (GW 11/00)

- Wolnoć Tomku w swoim domku. 
- Wolnoć Tomku... (GW 75/01)

- Wolnoć Tomku w swoim... (GW 30/02)

Manche Sprichwörter scheinen demnach als Material für die Artikelüberschriften besonders beliebt zu sein und werden in den unterschiedlichsten Modifikationen, meistens mit dem reduzierten zweiten Teil, immer wieder verwendet, obwohl sie von den Minima nicht erfasst worden sind. Das kann einerseits an der strukturellen Eignung des Sprichwortes selbst liegen, aber auch daran, dass es gerade durch die mehrmalige Verwendung an exponierten Textstellen (wieder) bekannt geworden ist und diese Bekanntheit in weiteren Texten genutzt wird.

Eine solche fast serienhafte Verwendung weist das aus dem 18. Jahrhundert stammende polnische Sprichwort Polak, Wegier - dwa bratanki, $i$ do szabli i do szklanki auf, das in diverser Gestalt in Überschriften verschiedener Pressetitel schon über mehrere Jahre erscheint:

- Oby nie jak bratanki (GW 160/04) [Die Ungarn haben Probleme mit der Nutzung der EU-Gelder, die Polen sollen daraus lernen]

- Polish, English dwa bratanki (NP 1/06) [Englischunterricht soll in der Schule ab dem 7. Lebensjahr beginnen]

- PiS, Platforma - dwa bratanki (P 48/06) [Die beiden Parteien stehen sich trotz ständiger Streitereien programmatisch nahe]

- Dwa bratanki (P 39/06) [Aktuelle Situation in Ungarn]

- Polak, Niemiec - dwa bratanki (W 21/06) [Różewicz und Grass treffen sich auf der Buchmesse in Warszawa]

- Polak, Czech - dwa bratanki ( W 9/07) [gute Beziehungen zwischen den Präsidenten der beiden Länder]

- Polak, Niemiec dwa bratanki (GW 150/07) [Ergebnisse einer Umfrage zeigen, dass beide Nationen ihre Beziehungen positiv einschätzen]

- Polak, Masaj-dwa bratanki! (A 1/07) [Menschen mit verschiedener Hautfarbe können sich genetisch ähnlich sein]

- Polak, Chińczyk - dwa bratanki (A 17/08) [Die Polen reisen gern nach China und stellen viele Gemeinsamkeiten im Benehmen fest]

Die Reduktion auf den ersten Teil verbunden mit dem Austausch des zweiten Lexems, wodurch jedesmal eine neue, manchmal recht unerwartete Opposition zu Polak entsteht, oder die Verneinung der Aussage des ersten Teiles lassen beim Leser Neugier auf den Artikel entstehen und stimmen ihn auf die Themenentfaltung ein. Die Umformulierung kann ihm sogar als „Interpretationshilfe zum nachfolgenden Text" (Hellwig 1984: 6) dienen, was der Beleg mit der Verneinung wohl am besten illustriert. Da das Sprichwort von dem polnischen Minimum nicht erfasst ist, sich aber als Material für Überschriften in der Presse geeignet erwiesen hat, kann 
vermutet werden, dass es seine neue Bekanntheit gerade der Verwendung in der Presse verdankt und somit zu einer Renaissance gelangen kann.

In den an ein beleseneres Publikum adressierten Pressetiteln lassen sich in den Überschriften vereinzelt auch Sprichwörter im lateinischen Wortlaut finden, manche davon in sprachspielerisch modifizierter Form. Die Belege unten illustrieren eine Reduktion des zweiten Teils, eine auf phonetischer Ähnlichkeit basierende Veränderung des lateinischen Lexems dura zu einem polnischen Lexem, womit es zu einer dennoch sinnvollen optisch sichtbaren Verflechtung des Textes kommt und einen geschickten Einschub polnischer Lexeme in den lateinischen Wortlaut darstellt, so dass eine Verneinung des ursprünglichen lateinischen Sinnes entsteht, die an dem neuen Wortlaut auch visuell deutlich zu erkennen ist. Das Verstehen und Erkennen des Sinnes der so modifizierten Sprichwörter setzt ein gutes Allgemeinwissen, die Kenntnis der aktuellen Ereignisse und sprachliche Reflexion voraus.

- Vox populi (W 41/98) [Zur Rolle der öffentlichen Meinung]

- Roma locata, ale causa nie finita (P 45/04) [Die Verfassung der EU ist zwar in Rom unterzeichnet worden, doch sie muss noch von allen Ländern ratifiziert werden, so dass der Prozess noch nicht abgeschlossen ist]

- Bzdura lex, sed lex (W 27/04) [Der polnische Sejm verabschiedet schlecht konstruierte, unlogische Gesetze]

Mieder zeigt in seinen Ausführungen, dass gerade in der Mediensprache Sprichwörter gern benutzt werden, die nicht die traditionelle biblische bzw. griechisch-lateinische Herkunft aufweisen, und er belegt das mit dem aus dem Türkischen stammenden Sprichwort Hunde bellen, aber die Karawane zieht weiter, das auch im Englischen wie im Polnischen eine analoge Entsprechung hat (vgl. Mieder 2004: 51).

- Die Karawane zieht weiter (DS 15/06) [späte Kritik an Altbundeskanzler G. Schröder]

- Psy szczekaja, karawanna PSL idzie dalej (GW 106/08) [Die Bauernpartei betreibt trotz kritischer Stimmen ihre alte Kaderpolitik]

- Karawana jedzie dalej. Tylko dokad (GW 144/08) [Situation in der UE nach dem Nein von Irland zum Lissaboner Vertrag]

Dieses Sprichwort ist von keinem der Minima erfasst, dafür aber ist das oben belegte Zeit ist Geld, das auf den Amerikaner Benjamin Franklin und seinen Ratschlag Time is money zurückgeht (vgl. Bötcher 1982: 328), in das älteste deutsche Sprichwortminimum aufgenommen worden (vgl. Mieder 1992: 16).

5. Alle diese Belege zeigen, dass der Kreativität im Umgang mit Sprichwörtern als Überschriften im Prinzip keine Grenzen gesetzt sind. Die vorgenommenen Modifikationen dienen dabei nicht nur dazu, den Leser aufmerksam zu machen und zu 
intrigieren, sondern auch, um das Sprichwort in einen inhaltlichen Bezug zu dem Artikel zu bringen, wobei manchmal ein einziges Lexem dazu genügt. Ausgefallene Modifikationen können bei anspruchsvolleren Lesern einen zusätzlichen Reiz entstehen lassen und sie zum Mitmachen bei der Interpretation auffordern. Einem Teil der Leser kann allerdings der Sinn der Abwandlung verborgen bleiben, der Artikel als solcher wird dennoch verstanden. Während der Lektüre kann sich auch die Nutzung des Sprichwortes in der Überschrift klären, wenn im Text auf dieses nochmals eingegangen wird. Der Autor der Überschrift muss jedoch auch damit rechnen, dass der Sinnbezug zwischen der Überschrift und dem Ko-Text nicht erkannt wird und das in seine Textarbeit einkalkulieren. Die Nutzung von allgemein bekannten Sprichwörtern aus den Minima garantiert daher eher, dass diese erkannt und die an ihnen vorgenommenen Modifikationen sowie der Sinnbezug zum Artikel schneller entdeckt werden. Für die Arbeit an der Erforschung des Bekanntheitsgrades und der Erstellung aktueller Sprichwortminima ist das ein zusätzlicher Beweis ihrer Nützlichkeit.

\section{Literatur}

Baur, Rupprecht, S.; Chlosta, Christoph; Grzybek, Peter (1996): Das Projekt 'SprichwörterMinima im Deutschen und Kroatischen': What is worth doing - do it well“. In: Muttersprache 2, 162-189

Baur, Rupprecht, S.; Chlosta, Christoph (1996): „Welche Übung macht den Meister?“ Von der Sprichwortforschung zur Sprichwortdidaktik. In: Fremdsprache Deutsch. Heft 15, 17-24

Bötcher, Kurt, et al. $\left({ }^{2} 1982\right)$ : Geflügelte Worte. Lepzig

Chlosta, Christoph; Grzybek, Peter (2004): Was heißt eigentlich „Bekanntheit“ von Sprichwörtern? Methodologische Bemerkungen anhand einer Fallstudie zur Bekanntheit angloamerikanischer Sprichwörter in Kanada und in den USA. In: C. Földes (Hg.): Res humanae proverbiorum et sententiarum. Ad honorem Wolfgangi Mieder. Tübingen, 37-57

Di Meola, Claudio (1998): Schlagzeilen in Presse und Werbung. In: Deutsche Sprache 26, 218-239

Grzybek, Peter (1991): Sinkendes Kulturgut? Eine empirische Pilotstudie zur Bekanntheit deutscher Sprichwörter. In: Wirkendes Wort 2. 239-264

Hellwig, Peter (1984): Titulus oder über den Zusammenhang von Titeln und Texten. Titel sind ein Schlüssel zur Textkonstitution. In: Zeitschrift für germanistische Linguistik 12, 1-20

Kim, Kun Hwam (1999): Deutsche Sprichwörter im Spiegel der Gesellschaft. Analyse der von Deutschen am häufigsten genannten Sprichwörtern. In: Zeitschrift für deutsche Philologie $118,87-102$

Litovkina, Anna T.; Mieder, Wolfgang (2006): Old Proverbs Never Die, They Just Diversify. A Collektion of Anti-Proverbs. Burlington, Veszprém

Lüger, Heinz-Helmut $\left({ }^{2} 1995\right)$ : Pressesprache. Tübingen

Mieder, Wolfgang (1973): Verwendungsmöglichkeiten und Funktionswerte des Sprichwortes in der Wochenzeitung. In: Muttersprache, Jg. 83, 89-101

Mieder, Wolfgang (1992): Sprichwort - Wahrwort!? Studien zur Geschichte, Bedeutung und Funktion deutscher Sprichwörter. Frankfurt am Main

Mieder, Wolfgang (1997): Verkehrte Worte. Antizitate aus Literatur und Medien. Wiesbaden 
Mieder, Wolfgang (2004): „Andere Zeiten, andere Lehren“ - Sprach- und kulturgeschichtliche Betrachtungen zum Sprichwort. In: Kathrin Steyer (Hg.), Wortverbindungen - mehr oder weniger fest. Berlin/New York, 415-438

Nord, Christiane (1993): Einführung in das funktionale Übersetzen. Tübingen

Prędota, Stanisław (1996): Modyfikacje parodystyczne przysłów (na materiale języka niderlandzkiego). In: Andrzej M. Lewicki (Hg.), Problemy frazeologii europejskiej, I. Warszawa, 29-37

Sandig, Barbara (1971): Syntaktische Typologie der Schlagzeile. Möglichkeiten und Grenzen der Sprachökonomie im Zeitungsdeutsch. München

Sandig, Barbara (1986): Stilistik der deutschen Sprache. Berlin

Singer, Samuel (1944-1947): Sprichwörter des Mittelalters. 3 Bände, Bern

Szpila, G. (2002): Minimum paremiologiczne języka polskiego - badanie pilotażowe. In: Język Polski LXXXII/1, 36-42

Umurova, Gulnas (2005): Was der Volksmund in einem Sprichwort verpackt ... Moderne Aspekte des Sprichwortgebrauchs anhand von Beispielen aus dem Internet. Bern

Textquellen mit Siglen:

$$
\begin{aligned}
\mathrm{A} & =\text { Angora } \\
\mathrm{DS} & =\text { Der Spiegel } \\
\mathrm{GW} & =\text { Gazeta Wyborcza } \\
\mathrm{NP} & =\text { Newsweek Polska } \\
\mathrm{P} & =\text { Polityka } \\
\mathrm{S} & =\text { Stern } \\
\mathrm{TVT} & =\text { TVToday } \\
\mathrm{W} & =\text { Wprost }
\end{aligned}
$$

